\title{
MAPEAMENTO PARTICIPATIVO DE RISCOS PERCEBIDOS POR PESCADORES (AS) DE COMUNIDADES TRADICIONAIS NA ZONA NORTE DA BAÍA DE TODOS OS SANTOS
}

\author{
Erika Silva Vieira Serbeto ${ }^{1}$; Fábio Pedro Souza de Ferreira Bandeira ${ }^{2}$; Luciana Almeida \\ dos Santos ${ }^{3}$; Raiza Rabelo Ramos Almeida ${ }^{4}$ e Brenda Elaine Silva de Jesus ${ }^{5}$ \\ 1. Bolsista PIBIC/FAPESB, Graduanda em Ciências Biológicas, Universidade Estadual de Feira de Santana, e-mail: \\ erikaserbeto20@gmail.com \\ 2. Orientador e coordenador do Núcleo de Pesquisa em Ambiente, Sociedade e Sustentabilidade (NUPAS), \\ Departamento de Ciências Biológicas, Universidade Estadual de Feira de Santana, e-mail: fpbandeira@ gmail.com \\ 3. Participante do NUPAS, Universidade Estadual de Feira de Santana, e-mail: thluciana@gmail.com \\ 4. Bolsista PROBIC/UEFS, Participante do NUPAS, Departamento de Ciências Biológicas, Universidade Estadual de \\ Feira de Santana, e-mail: raizarabelo96@gmail.com \\ 5. Bolsista PIBIC/CNPq, Participante do NPAS, Departamento de Ciências Biológicas, Universidade Estadual de \\ Feira de Santana, e-mail: brenda_.e@ hotmail.com
}

PALAVRAS-CHAVE: vulnerabilidade, indústria petrolífera, pescadores artesanais

\section{INTRODUÇÃO}

A Baía de Todos os Santos (BTS) é considerado o segundo maior acidente geográfico no Brasil, perdendo apenas em dimensão à Baía de São Marcos, no Maranhão, possuindo uma superfície de $1.233 \mathrm{~km}^{2}$ (CRA, 2001). Ela pode ser compreendida como um grande complexo estuarino que conta com as contribuições significativas de rios do porte do Paraguaçu, da Dona, Subaé e Jaguaripe, além muitos outros de menor porte que deságuam em seu interior (BRITO, 2001, p.98). As regiões que contornam a BTS enfrentam, atualmente alguns desafios de delimitações concretas, além de desafios ocasionados pela intensificação do seu potencial turístico, que vem crescendo ao longo das décadas, bem como as novas formas de relação dessas regiões com a capital. A região também é bastante impactada por mudanças socioambientais marcantes em decorrência dos processos de industrialização e urbanização que só vêm se intensificando desde os 60s (BANDEIRA et al., 2009). Assim, existe uma carência de estudos com abordagens que tratem da percepção das comunidades e povos tradicionais a respeito dos riscos aos quais eles estão expostos bem como de abordagens que tratem a multidimensionalidade da sua percepção (MARANDOLA JR e HOGAN, 2004), uma vez que essas comunidades dependem diretamente dos ecossistemas e recursos naturais da baía para sua reprodução sociocultural. Partindo dessa realidade, o presente estudo teve como objetivo mapear de maneira participativa os riscos percebidos por membros de comunidades pesqueiras residentes na porção norte da Baía de Todos os Santos, em particular, do município de Madre de Deus.

\section{MATERIAL E MÉTODOS}

Primeiramente, tentou-se o contato nas comunidades por meio de associações/colônia de pescadores ou morados para a obtenção da autorização para a realização da pesquisa. Após reunião para esclarecimento e autorização da pesquisa, se realizou a mobilização da comunidade para as oficinas. Na primeira oficina foi discutido, juntamente com o grupo participante, os pescadores e pescadoras que atenderam à mobilização, a maneira como eles identificavam o próprio território, apresentando para isso uma imagem de satélite recente do município. Então, os participantes foram divididos em cinco grupos, de acordo com o critério deles, para a produção dos etnomapas. Os participantes da oficina utilizaram papel manteiga sobreposto a uma imagem de satélite da região e com o auxílio de giz de cera, lápis de cor e hidrocores eles indicaram nos mapas os locais de 
pesca, riscos, recursos pesqueiros e áreas de acesso restrito aos pescadores por atividades industriais. Posteriormente foi realizada uma outra oficina onde foi trabalhado de maneira coletiva, sem a divisão de grupos, onde eles listaram os riscos existentes na região que atrapalham as atividades de pesca e classificaram esses riscos de acordo com sua gravidade. Para a classificação dos riscos usamos uma abordagem similar à de Santos (2013), onde utilizamos três cores: vermelho (muito grave), preto (grave) e azul (pouco grave). Por fim foi montado uma linha do tempo dos acontecimentos que eles se lembravam e julgavam importantes.

\section{ANÁLISE E DISCUSSÃO DOS RESULTADOS}

A primeira oficina contou com a participação de 26 pessoas, sendo sua maioria pescadoras. Foram organizados cinco grupos e cada grupo recebeu uma imagem de satélite da área para trabalhar. Cada grupo percebeu o território de um modo diferente, porém todos identificaram a maioria dos elementos e/ou localidades, sendo que um grupo não lembrava ou não julgava necessário colocar no mapa, o outro grupo indicava. Assim, os etnomapas produzidos pelos diferentes grupos se complementaram. No geral, percebemos que os grupos identificaram áreas em comum (as relacionadas à atividade da pesca e às instalações da Petrobras), porém cada grupo apresentou um nível de detalhamento distinto no seu etnomapa (figura 1). Isso demonstra como a percepção do espaço e do ambiente está relacionada com a vivência do indivíduo, o que pode ser ampliado para análise dos grupos. No segundo dia de oficina contou-se com a presença de 56 participantes e novamente de maioria feminina. No primeiro momento os presentes listaram os riscos que dificultavam as atividades de pesca. Os riscos citados foram: (1) a presença da Petrobras, (2) as tubulações (PP4), (3) os vazamentos de produtos (óleo e gás), (4) o excesso de ruído da refinaria, (5) o cheiro forte de enxofre, (6) pesca predatória com bomba, (7) o esgoto da Petrobras e das casas, (8) o lixo, (9)as doenças trazidas por turistas e a tripulação dos navios, (10) poluição gerada pelo turismo, (11) água de lastro dos navios (12) a restrição aos locais de pesca pelas presença das embarcações, (13) os perigos intrínsecos à atividade da pesca, (14) os temporais que trazem sujeira e (15) a poluição sonora urbana. Em um segundo momento, os participantes classificaram a gravidade de cada risco citado anteriormente. Para essa classificação foram utilizadas três cores: vermelho (muito grave), preto (grave) e azul (pouco grave). Para a grande maioria dos riscos citados houve o consenso quanto a classificação, porém em alguns houveram divergências e, nesses casos, acabou-se utilizando duas classificações para um mesmo risco (figura 2). Dos quinze riscos citados pela comunidade oito deles estão associados com as atividades petroquímicas que são desenvolvidas na região. A instalação da indústria petrolífera no Recôncavo, em especial no município de Madre de Deus, vem promovendo no decorrer dos anos diversas alterações espaciais, políticas, econômicas, estéticas, culturais e ecológicas, além de outras, que representam, em maior parte, o aumento e/ou origem de conflitos e vulnerabilidades no território (FREIRE, 2011). A poluição petroquímica da água, segundo Hatje \& Andrade (2009), é identificada na BTS, principalmente, nas localidades que estão próximas ao Centro Industrial de Aratu, que fica na Região Metropolitana de Salvador. Nessa região é operante o Porto de Aratu, assim como empreendimentos de seguimento químico, de minerais, metalúrgicos, fertilizantes, entre outros. A maior preocupação com esse tipo de poluição é a contaminação química dos mariscos que são consumidos e comercializados pelas comunidades não só de Madre de Deus, mas por todas as populações que residem no entorno da BTS e dependem dos seus recursos, então, essa questão se torna central para a saúde pública. Praticamente todos os riscos receberam a classificação de muito grave, isso provavelmente se deva ao fato de que tais riscos dificultam ou impedem diretamente o desenvolvimento das 
atividades econômicas dos pescadores (as), bem como se relacionam com questões que ameaçam a saúde e até mesmo apresentam ameaça à vida da população. Nos casos nos quais houveram duas classificações, como os perigos da pesca, se deve ao fato de que a gravidade desse risco varia de acordo com as estações, segundo os relatos dos participantes. Quanto aos eventos evidenciados na linha do tempo, vemos que são eventos que interferiram e ainda interferem diretamente nas atividades de pesca. Pudemos observar que todos os problemas socioambientais evidenciados ocorreram após instalação da indústria petrolífera no município (figura 3). Além dos acidentes relacionados a essa indústria, os participantes da pesquisa destacaram que quando passaram a ter a carteira de pesca, que é um marco importante para as comunidades pesqueiras, pois reconhece e oficializa a profissão de pescador; o direito ao defeso do camarão nos períodos de desova (abril/maio e setembro/outubro) e o início das dificuldades em receber o defeso com a mudança dos órgãos responsáveis pelo pagamento.

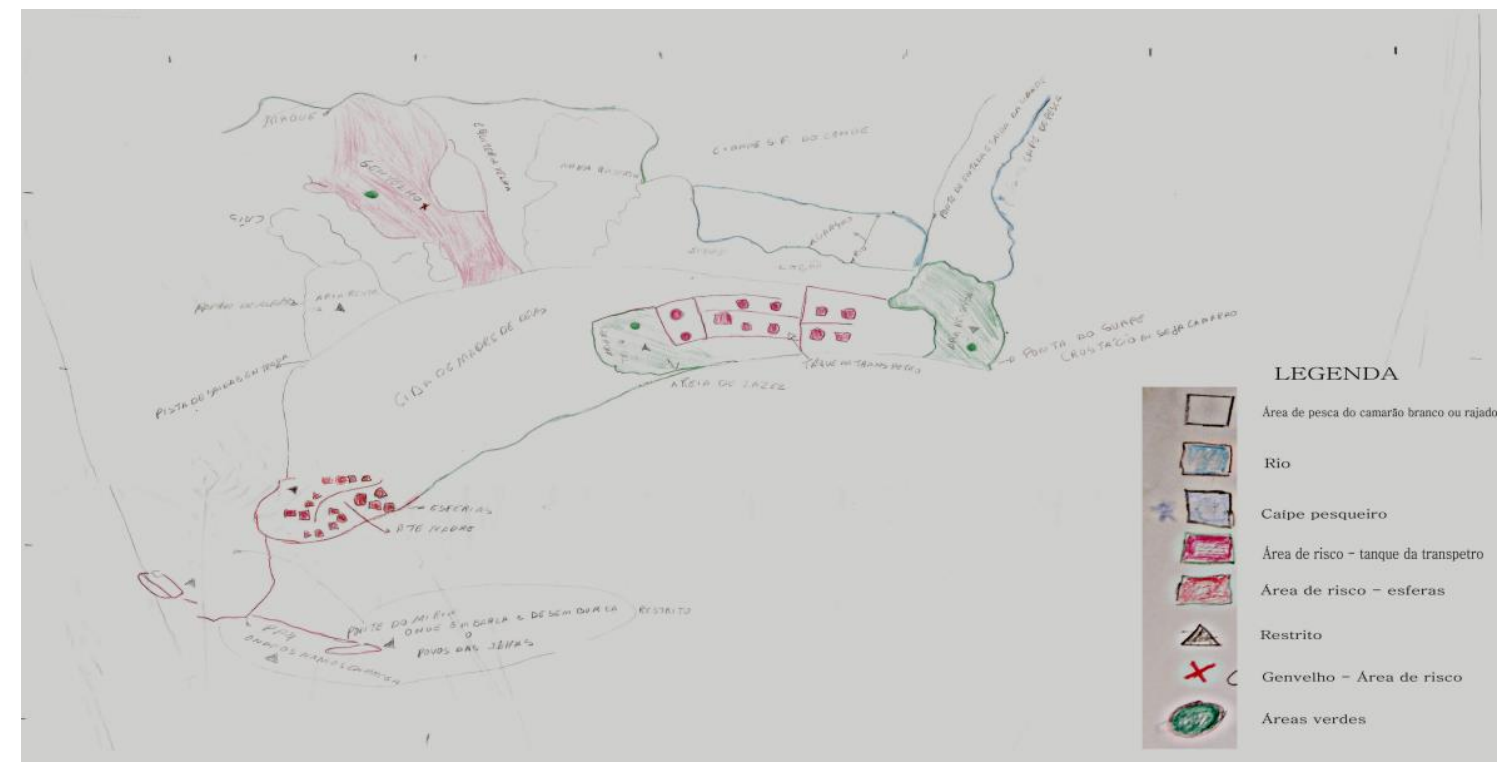

Figura 1: Etnomapa produzido por um dos grupos de pescadores e pescadoras da Associação Central da Pesca, na oficina 1, Madre de Deus, Ba, 20018

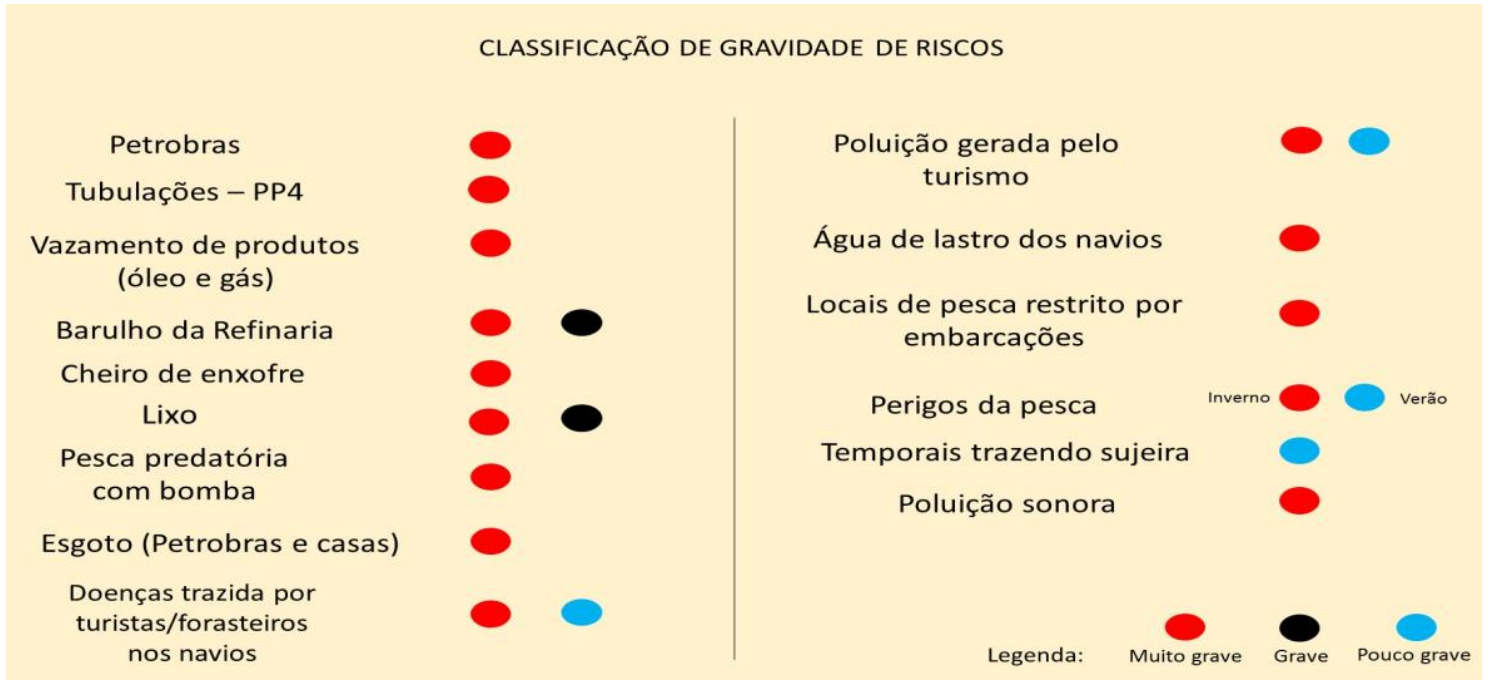

Figura 2: Riscos citados pelos pescadores e pescadoras da Associação Central da Pesca, participantes da oficina 2 e a classificação de sua gravidade de acordo com os mesmos, Madre de Deus, Ba., 2018. 


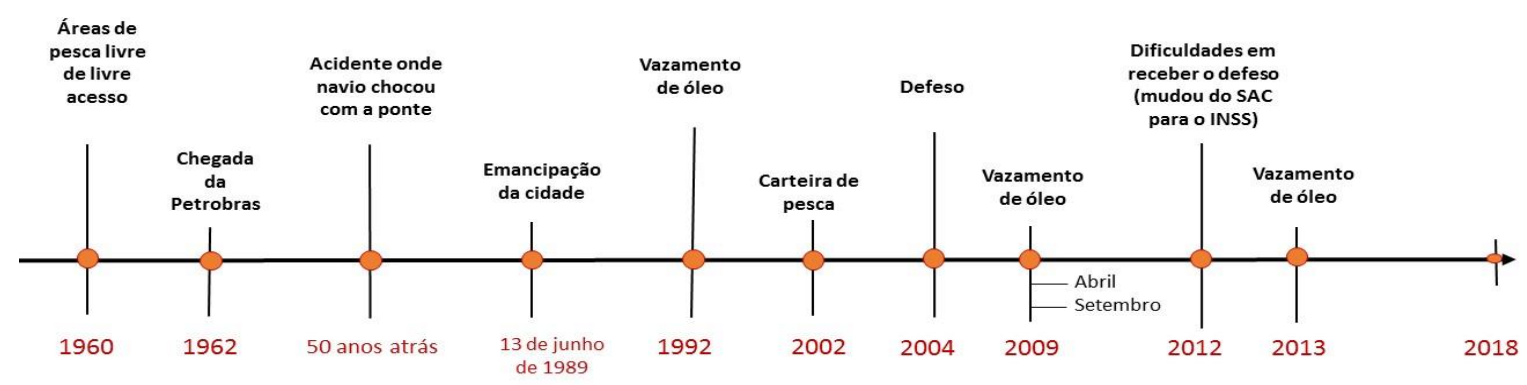

Figura 3: Linha temporal organizada com os pescadores e pescadoras da Associação Central da Pesca, na oficina 1, Madre de Deus, Ba, 2018.

\section{CONSIDERAÇÕES FINAIS}

Mesmo inseridos dentro de um mesmo grupo, o de comunidades pesqueiras artesanais, os indivíduos percebem o território de modo diferente e tal fato tem relação com as suas vivências pessoais e acontecimentos relevantes para os mesmos. Os principais riscos citados pelos participantes da pesquisa então diretamente ligados às atividades petroquímicas na região, que afetam tanto o desenvolvimento de suas atividades laborais, bem como traz a sensação de insegurança e vulnerabilidade para a população. Quase todos os riscos citados foram classificados como muito graves e tal fato corrobora as afirmações anteriores, assim como os eventos que eles julgaram importantes para eles nos últimos anos. Assim, com essa pesquisa podemos verificar que os modos de vida e as atividades laborais, influenciam na percepção de risco de cada indivíduo em uma comunidade pesqueira. Estes resultados indicam que é necessário que os planos de gerenciamento de risco na BTS considerem o conhecimento e percepção das comunidades tradicionais e residentes, para que seja de fato efetivo.

\section{REFERÊNCIAS}

CRA. 2001.Avaliação das águas costeiras superficiais, BTS Praias de Salvador. Salvador/BA.

BANDEIRA, F. P. et al. 2009. Estudo etnoecológico sobre a percepção das populações ribeirinhas dos riscos e impactos ambientais na Baía de Todos-os-Santos (BTS) / Fábio Pedro S. de F. Bandeira (org.). - Salvador: Instituto do Meio Ambiente, Secretaria do Meio Ambiente e Recursos Hídricos; EGBA,. 137 p. : il. No prelo

BRITO, R. R. C. de. 2001. A gestão da Baía de Todos os Santos. BAHIA ANÁLISE \& DADOS v.11, n.2, p.98-100.

FREIRE, J. T. 2011. A percepção de riscos ambientais tecnológicos no município de Madre de Deus - Ba. Grupo Costeiros. Universidade Federal da Bahia. Salvador.

HATJE, V.; ANDRADE, J.B. 2009. Contaminação química. In: HATGE, V; ANDRADE, J.B. Baía de Todos os Santos: aspectos oceanográficos. Salvador: EDUFBA, p. 19-22.

MARANDOLA JR., E.; HOGAN, D. J. 2004. O risco em perspectiva: tendências e abordagens. Geosul, Florianópolis, n. 38.

SANTOS, Andréa Iridan dos. 2013. Modelagem etnoecológica da percepção de vulnerabilidades, riscos e impactos socioambientais em comunidades quilombolas da Baía de Todos os Santos. [manuscrito] Dissertação de Mestrado / Andrea Iridan dos Santos. Feira de Santana. 